\title{
A Rare Variant of Capgras Syndrome in Alzheimer's Disease
}

\author{
Corinne Fischer, Annie Keeler, Luis Fornazzari, Lee Ringer, Tawnya Hansen, Tom A. Schweizer
}

Can. J. Neurol. Sci. 2009; 36:509-511

Alzheimer's disease (AD) may occasionally be complicated by a rare but characteristic misidentification delusion referred to as "Capgras Syndrome". Capgras syndrome was first described nearly a century ago, and was termed "illusion des sosies" (illusion of doubles) ${ }^{1}$. The other types of delusional misidentifications include Fregoli syndrome, the syndrome of subjective doubles, and the syndrome of intermetamorphosis, which involves a person dressing in disguise as several other people, a duplicate self, and exchanging identities of people in a particular setting ${ }^{2}$. None of these syndromes address the duplication of objects or replacement of objects with exact replicas, although there have been case reports of this phenomenon occurring in various neurological disorders. To our knowledge this is the only case report to look at this phenomenon in a patient with documented AD.

There have been several case reports involving delusional misidentification of inanimate objects. One of the first cases was reported in 1977 and involved a 43-year-old woman with schizophrenia who believed that both her husband and his belongings had been replaced by exact replicas ${ }^{3}$. At least six other cases of object duplication have been reported in schizophrenia ${ }^{4-7}$. Cases have also been reported to occur during an acute psychotic episode ${ }^{8}$ and during a major depressive episode $^{9}$. In 1990 a correspondence written by Anderson described an 84-year-old woman with possible AD who insisted her house and its contents had been replaced by identical objects ${ }^{10}$. However, no neuropsychological testing or structural brain imaging was done to confirm the diagnosis. Three cases of this rare form of Capgras syndrome were reported in 1994. The authors coined the term "delusional gross replacement of inanimate objects"11. The most recent case described a woman with generalized seizures who believed that her household belongings and personal possessions were being replaced in order to transmit illness and ultimately cause her death ${ }^{12}$. We report a case of a patient with documented $\mathrm{AD}$ who presented with Capgras syndrome involving inanimate objects.

\section{Case Report}

An 87-year-old woman was brought to a local emergency department for assessment of cognitive and behavioural changes. For approximately one month prior to admission she held a distressing belief that a woman, whom she did not know or recognize, had been entering her apartment and stealing her belongings. She began to notice that if nothing had been stolen overnight, one or more of her expensive porcelain figurines would have been swapped for cheap imitations. At the time of her admission, she explained that over three-quarters of her

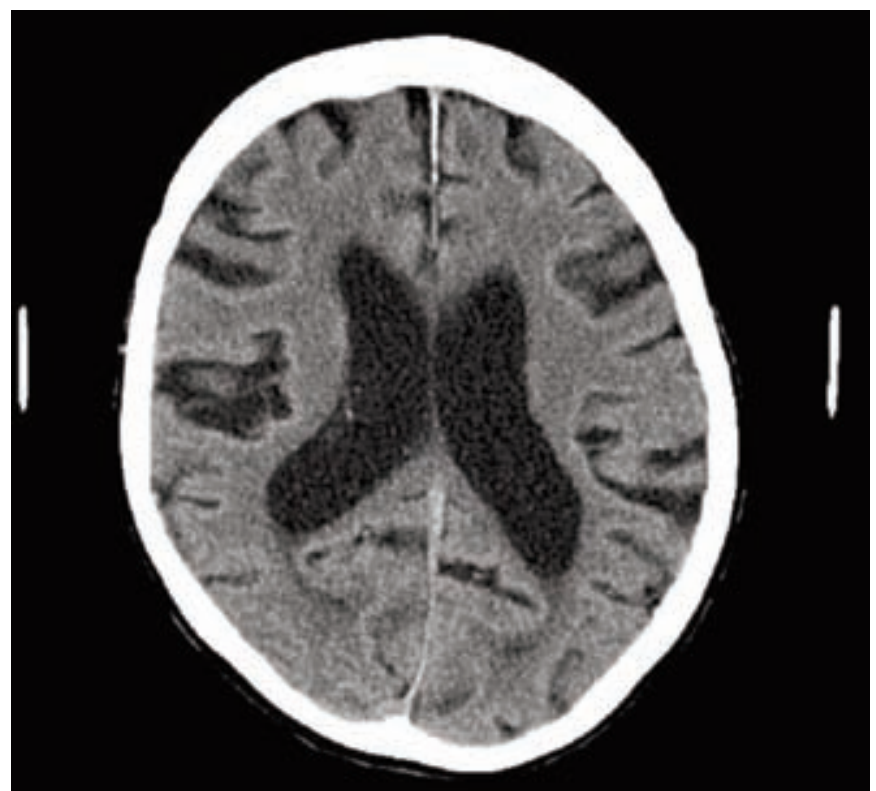

Figure: CT Scan of Brain.

figurines and many of her expensive plates had been swapped in such a fashion. Although the patient was quite upset with respect to these replacements, she had no explanation as to how or why this woman was coming into her apartment. No other delusional beliefs were elicited and she denied any hallucinations, thought broadcasting, thought insertion or ideas of reference. She also denied any depressive or anxiety symptoms. She admitted to some mild loss of memory but thought that this could be age related and denied any change in daily functioning as a result.

The patient's past medical history was significant for colon cancer with resection and reversal of colostomy (2002),

From the University of Toronto (CF, AK, LF, LR, TH, TAS); Mental Health Service (CF, LR), Department of Neurology (LF), Department of Neurosurgery (TAS), Keenan Research Centre of the Li Ka Shing Knowledge Institute (CF, TAS), St. Michael's Hospital, Toronto, Ontario, Canada.

Received December 8, 2008. Final Revisions Submitted January 23, 2009 Correspondence to: Corinne Fischer, \#17044 Cardinal Carter Wing, St. Michael's Hospital, \#30 Bond Street, Toronto, Ontario, M5B 1W8, Canada. 
hyperlipidemia, hypertension, cholecystitis, small bowel obstruction, osteoporosis, fractured left distal radius (2003) and fractured patella (1995). Her medications on admission included Atenolol $50 \mathrm{mg}$ p.o. once a day, Citalopram $10 \mathrm{mg}$ daily, Lansoprazole $30 \mathrm{mg}$ a day, Ranitidine $150 \mathrm{mg}$ b.i.d, Tylenol 650 mg q. 4-6h. p.r.n. and Gravol p.r.n. The Gravol and Tylenol were started while in hospital for mild abdominal pain but were rarely used. Her Ranitidine was also discontinued on admission. The other medications were started prior to her coming into hospital, but it is unclear when.

Her family described a one-year history prior to admission of progressive memory loss, mild difficulty finding words and some difficulties with executive function. However, the patient did not have any formal testing of her memory, including neuropsychological testing, prior to admission and did not have any prior medical admissions relating to cognitive impairment. Routine physical examination showed no obvious abnormalities. Screening neurological exam was within normal limits and there was no evidence of significant visual or hearing impairment. The patient was seen by occupational therapy during her admission. Based on observation and collateral history obtained from her nephew, she was independent with all of her routine activities of daily living with the exception of bathing, for which she required some supervision. In terms of her instrumental activities of daily living, she was noted to have problems with paying her bills, buying her groceries and maintaining her household for one year prior to admission.

Global cognitive testing revealed a Mini-Mental Status Examination score of 23/30, which was repeated a day later with the same score. The patient also had a Montreal Cognitive Assessment score of $15 / 30$. Structural brain imaging using computerized tomography was done during her admission (see Figure) and demonstrated old lacunar infarcts bilaterally in the caudate and lentiform nucleus, mild microangiopathic changes and age appropriate generalized cortical atrophy. However, she had no clinical history of stroke. A single photon emission computer tomography (SPECT) scan revealed reduced uptake in the left temporal-parietal lobe. Based on the clinical findings, brief cognitive testing and neuroimaging the diagnosis of mixed (Alzheimer's-vascular) dementia was made approximately one week into the patient's hospitalization. Treatment with an acetylcholinesterase inhibitor was deferred as the patient was not interested and deemed competent to refuse. Risperidone $0.25 \mathrm{qhs}$ was initiated but was stopped after 24 hours as it seemed to worsen her cognitive status. No other therapies were pursued, as she did not seem overly distressed by the delusion. A diagnosis of Dementia with Lewy Bodies (DLB) was considered based on the prominence of the delusions and the finding of neuroleptic sensitivity. However, it was later excluded based on the neuroimaging results and the absence of parkinsonism on physical exam.

\section{Discussion}

Capgras syndrome is a behavioural phenomenon involving the delusional belief that a close relative, often a spouse, is an imposter. This delusion is quite common in $\mathrm{AD}$, with a prevalence ranging from $7 \%$ to $10 \%{ }^{13,14}$, although it may be associated with all forms of dementia. Rare forms of delusional misidentification, such as the case described, have been estimated to account for approximately $10 \%$ of all cases of Capgras syndrome ${ }^{15}$. However, the literature does not seem to reflect this estimate. It is hard to know whether or not cases of this rare form of delusional misidentification are not documented or whether they have been subsumed under another phenomenological term such as reduplicative paramnesia.

There are a number of interesting features that set this case apart from previously documented cases. One aspect is the lack of a persecutory feeling associated with the delusion. One potential explanation for the lack of this element in the current case may be that delusions in $\mathrm{AD}$ tend to fluctuate in intensity over time and it is possible that we may have been capturing her at a particularly good time. This is one reason why such symptoms should be followed longitudinally ${ }^{16}$.

Another interesting difference seen in this case is the coexistence of the belief that someone is stealing from her apartment. The belief that someone is stealing is a very common delusion in $\mathrm{AD}^{16}$ and tends to occur earlier in the disease course while delusions such as Capgras syndrome tend to occur later ${ }^{16}$. Yet our patient had mild AD. One potential explanation for this discrepancy may be that this rare variant of Capgras syndrome tends to occur earlier in disease course, or perhaps that it is an entity of its own.

In the current case, the patient's SPECT scan reveals decreased uptake in the left temporoparietal lobe. It has been found that in delusional AD patients ${ }^{17}$, and in interictal psychosis $^{18}$ with Capgras syndrome, there is decreased metabolism in the right temporal lobe. Another study looking at delusions of substitution related to an underlying organic etiology showed CT evidence of lesions in the left temporal lobe more frequently ${ }^{19}$. It is clear based on the lack of consensus in the literature that the neuroanatomical origins of this rare behavioural phenomenon have yet to be defined. However, there may be theoretical reasons why some parts of the brain are more likely to be involved than others. Alexander et al $(1979)^{20}$ in a single case report, suggested that "reduplicative paramnesia", which bears some resemblance to the phenomenon described, may stem from damage to multiple brain regions including the frontal lobes and the right hemisphere. Right hemisphere damage could disrupt visuospatial processing, leading to impaired recognition which can then not be reasoned out due to coexisting frontal lobe anomalies.

Capgras Syndrome can be seen in a variety of neurological disorders including $\mathrm{AD}$ and related dementias, epilepsy, acquired brain injury, cerebrovascular disease and drug abuse ${ }^{20}$. It has also been reported in schizophrenia, and schizoaffective disorder ${ }^{21}$. Approximately $81 \%$ of cases of Capgras syndrome occur in the presence of a neurodegenerative disorder ${ }^{19}$, suggesting it may be an important marker of cerebral damage. Recent publications on Capgras syndrome and dementia have attempted to identify the prevalence of this delusion in various subtypes of dementia. Harciarek et al $(2007)^{22}$ looked at the prevalence of misidentification delusions in a large Memory Clinic. He discovered that they tended to occur most commonly in patients with AD and DLB and much less commonly in patients with Frontal Temporal Dementia and Parkinson's Dementia. Similarly, Josephs et al $(2007)^{21}$ conducted a retrospective chart review of Capgras syndrome at a tertiary medical centre and discovered that it was most commonly associated with a 
neurodegenerative disease, but when this was not the case patients tended to be younger and have a history of stroke, substance abuse or schizophrenia.

There is a growing consensus that Capgras syndrome represents a form of delusional thinking in $\mathrm{AD}$. Much attention has been devoted in recent years to the study of delusions in AD. Capgras syndrome is perhaps one of the more rare subtypes. More common subtypes include delusions of theft, infidelity, etc. ${ }^{23}$ Delusions in AD have been shown to be associated with a number of adverse health outcomes, including premature institutionalization, increased mortality and increased caregiver burden $^{24}$. In the case of our particular patient, she did end up in a Long Term Care Home. It is hard to know the contribution of her particular symptom to this, although there is little doubt that her delusional thinking played a role. Recent research in this area has also linked the presence of psychosis to impaired frontal lobe function $^{25}$ and more rapid cognitive decline. Given the adverse consequences associated with the presence of Capgras delusions and other delusions in $\mathrm{AD}$, it is very important that we develop a better understanding of this complex symptom, both phenomenologically and neuroanatomically, in order to guide the development of more effective treatments.

\section{ACKNOWLEDGEMENTS}

The authors would like to acknowledge the contribution of the patient and their family to the publication of this article.

\section{FUNDING}

This article was made possible through a peer-reviewed grant obtained from the Alzheimer Society of Canada.

\section{REFERENCES}

1. Capgras JMJ, Reboul-Lachaux J. L'illusion des "sosies" dans un delire systematize chronique. Bulletin de la Societe clinique de medicine mentale. 1923; 11: 6-16.

2. Ellis HD, Luate JP, Rettersol N. Delusional misidentification syndromes. Psychopathology. 1994; 27: 1117-20.

3. Christodoulou GN. The syndrome of Capgras. Brit J Psychiat. 1977; 130: 556-64.

4. Enoch MD, Trethowan WH. The Capgras syndrome. Uncommon psychiatric syndromes. 2nd ed. Bristol: John Wright and Sons; 1979.

5. Green BH. Capgras syndrome. Brit J Psychiat. 1989; 154: 271.

6. Anderson DN. The delusion of inanimate doubles. Implications for understanding the Capgras phenomenon. Brit $\mathrm{J}$ of Psychiat. 1988; 153: 694-9.

7. Christodoulou GN. Syndrome of subjective doubles. Am J Psychiat. 1978; 135: 249-51.

8. Abed RR, Fewtrell WD. Delusional misidentification of familiar inanimate objects. A rare variant of Capgras syndrome. Br J Psychiat. 1990; 157: 915-17.

9. Rastogi SC. A variant of Capgras syndrome with substitution of inanimate objects. A rare variant of Capgras syndrome. Br J Psychiat. 1990; 156: 883-4.

10. Anderson DN. Understanding Capgras syndrome. Br J Psychiat. 1990; 157: 622-3.

11. Castillo PM, Berman CW. Delusional gross replacement of inanimate objects. Br J Psychiat. 1994; 164: 693-6.

12. Ghaffari-Nejad A, Toofani K. A report of Capgras syndrome with belief in replacement of inanimate objects in a patient who suffered from grandmal epilepsy. AIM. 2005; 8(2): 141-3.

13. Harwood DG, Barker WW, Ownby RL, Duara R. Prevalence and correlates of Capgras syndrome in Alzheimer's disease. Int J Geriatr Psych. 1999; 14: 415-20.
14. Jeste DV, Wragg RE, Salmon DP, Harris MJ, Thal LJ. Cognitive deficits of patients with Alzheimer's disease with and without delusions. Am J Psychiat. 1992; 149: 184-9.

15. Anderson DN, Williams E. The delusion of inanimate doubles. Psychopathology. 1994; 27(3-5): 220-5.

16. Fischer C. Delusions in Alzheimer's disease: a literature review. The Canadian Alzheimer Disease Review. April 2004: 11-15.

17. Geroldi C, Akkawi NM, Galluzzi S, Ubezio MC, Binetti G, Zanetti $\mathrm{O}$, et al. Temporal lobe asymmetry in patients with Alzheimer's disease with delusions. J Neurol Neurosurg Psychiatry. 2000; 69: $187-91$.

18. Horikawa H, Monji A, Sasaki M, Maekawa T, Onitsuka T, Nitzaka Y, et al. Different SPECT findings before and after Capgras' syndrome in interictal psychosis. Epilepsy Behav. 2006; 9(1): 189-92.

19. Signer SF. Localization and lateralization in the delusion of substitution. Capgras symptom and its variants. Psychopathology. 1994; 27(3-5): 168-76.

20. Alexander MP, Stuss DT, Benson DF. Capgras syndrome: A reduplicative phenomenon. Neurology. 1979; 29: 334-9.

21. Josephs KA. Capgras syndrome and its relationship to neurodegenerative disease. Arch Neurol. 2007; 64(12): 1762-6.

22. Harciarek M, Kertesz A. The prevalence of misidentification syndromes in neurodegenerative diseases. Alz Dis Assoc Disord. 2008; 22: 163-9.

23. Migliorelli R, Petracca G, Teson A, Sabe L, Leiguarda R, Starkstein SE, et al. Neuropsychiatric and neuropsychological correlates of delusions in Alzheimer's disease. Psychol Med. 1995: 25(3); 505-13.

24. Levy ML, Cummings JL, Fairbanks LA, Masterman D, Miller BL, Craig $\mathrm{AH}$, et al. Longitudinal assesment of symptoms of depression, agitation, and psychosis in 181 patients with Alzheimer's disease. Am J Psychiatry. 1996: 135; 1438-43.

25. Mega MS, Lee L, Dinov ID, Mishkin F, Toga AW, Cummings JL, et al. Cerebral correlates of psychotic symptoms in Alzheimer's disease. J Neurol Neurosurg Psychiatry. 2000: 69(2); 167-71. 